\title{
A new experimental method for hiatal reinforcement using connective tissue patch transfer
}

\author{
A. Vereczkei, ${ }^{1}$ G. Varga, ${ }^{1}$ T. Tornoczky, ${ }^{2}$ A. Papp, ${ }^{1}$ Ö. P. Horvath ${ }^{1}$ \\ Departments of ${ }^{1}$ Surgery and ${ }^{2}$ Pathology, Medical School University of Pécs, Pécs, Hungary
}

SUMMARY. The closure of a large hiatal hernia still represents a challenge for the surgeon. Mesh reinforcement of a hiatoplasty generally decreases recurrence rate. An artificial mesh is cheaper compared with a biologic one, but has a higher complication rate. Our aim was to introduce a new biologic reinforcement method with less expenses. During organ donation for transplantation, tissue islets from pericardium and fascia lata were cryopreserved in a tissue bank. Later, the grafts were transplanted on the diaphragm of mongrel dogs. After 1, 3, and 6 months, the animals were sacrificed, and the transplanted patches were macroscopically and microscopically examined. There were no macroscopic signs of inflammation, abcedation, or significant adhesion formation. The grafts were well recognizable, with palpable thickening and moderate shrinkage. Microscopically, an organization process with fibrosis, neovascularization, and peritoneal integration could be observed. Reinforcement of a hiatoplasty with connective tissue transfer either with cryopreserved or autologous tissue is a good option. This is a cheap and easy method, which should also be tested in human interventions.

KEY WORDS: fascia lata, hiatal hernia, recurrence, reinforcement, tissue transfer.

\section{INTRODUCTION}

The closure of a large hiatal hernia still represents a challenge for the surgeon. Although there are papers about the successful closure of the hiatus with simple sutures, most of the studies report a $20-40 \%$ recurrence rate without mesh reinforcement. ${ }^{1}$

Our department has investigated this problem for a longer period using a biologic method. The reinforcement of the suture line after hiatal closure was successfully achieved using the patient's own teres ligament. ${ }^{2}$ However, the teres hiatoplasty is not feasible in every patient as it depends on the anatomical variations; thus, we tried to find a universal biologic solution that is applicable in every case and is preferably cheap.

We also have a transplantation unit, which routinely removes connective tissue during multiorgan donation, for orthopedic reconstructions. These tissues are stored deep frozen in a tissue bank for subsequent application. Because of this, an idea to use

Address correspondence to: Dr Andras Vereczkei, MD, PhD, Department of Surgery, Medical School University of Pécs,

Rákoczi ut 2, 7623 Pécs, Hungary.

Email: vereczkei@iseb.pote.hu connective tissue from the tissue bank for the reinforcement of a hiatal closure has arisen. We also had an experience with autologous fascia lata reinforcement of the lung parenchyma, with good results. ${ }^{3}$ However, there were no data in the literature about diaphragmatic application. Cryopreserved connective tissue has also never been applied before for hiatal reinforcement. Also, the size of an autologous fascia lata patch is limited by the incision, while a cryopreserved connective tissue patch can be much larger because at cadaver tissue harvest, the donor's aspect is de-emphasized in favor of a good transplantate. To obtain the required experience with this novel method, animal experiments were planned as a preliminary study to investigate conserved and deep frozen fascia lata and pericardium patches regarding their biologic behavior, scar tissue generation or resorption, and possible complications.

The animal studies were carried out with the approval of the local ethics committee.

\section{METHODS}

During multiorgan donations for the heart and orthopedic surgical departments, pericardial patches 
of $10 \times 10 \mathrm{~cm}$ and fascia lata patches of $7 \times 7 \mathrm{~cm}$ were removed. Grafts were treated with the conventional deimmunizing and conserving procedure for heart valves, which was accepted earlier and introduced by heart surgeons, and successfully used for a longer period. First, they were immersed into a preparative solution of $5^{\circ} \mathrm{C}$, containing $15 \mathrm{mg}$ amphotericin, $70 \mathrm{mg}$ cefuroxime, and $40 \mathrm{mg}$ clindamycin dissolved in $250 \mathrm{~mL}$ of Hank's solution (Sigma Aldrich, St. Louis, MO, USA). After 24-48 hours, the patches were transferred into the deimmunizing storage solution containing $25 \mathrm{~mL}$ of $\mathrm{DMSO}_{4}$ and $9 \mathrm{~mL}$ of $15 \%$ human albumin dissolved in $250 \mathrm{~mL}$ of Hank's solution. Samples were stored in the tissue bank freezer at $-78^{\circ} \mathrm{C}$.

Three months later, three mongrel dogs were narcotized and the cryopreservates from the tissue bank were defrosted. From an upper midline incision, 1-1 pericardial and fascia lata patches of $3 \times 3 \mathrm{~cm}$ were sutured to the muscular part of the left diaphragmatic arch. All patches were fixed at the four corners, and in the middle with $3 / 0$ polypropylene sutures, with decent spaces between them (Fig. 1). After the closure of the abdominal cavity, all animals were separated with special care until the primary healing of the wound without detecting any complications. Later, they were returned to their original places where they had a regular care. After 1, 3, and 6 months, 1-1 dog was narcotized again, and from a midline incision, the abdomen was explored, and the left diaphragmatic arch was excised for further analysis. All dogs were then terminated. The removed diaphragms were immersed into $6 \%$ formalin solution and transported to the department of pathology.

\section{RESULTS}

There were no intra- or postoperative complications; there were also no changes in the behavior, feeding, or weight of the animals. At the removal of the diaphragmatic arches, slight adhesions between the left liver lobe and the operative field were detected, but no suspicion of inflammation arose. The patches could be identified by the eyes; they remained in their initial position. There was a moderate shrinkage of the patches, with a palpable thickening of the tissue, where they were fixed (Fig. 2). As can be seen in the figure, the shrinkage was mainly at the sides, where no stitches were applied. Although it was not exactly measured, it was less than $20 \%$ at 3 months. At 6 months, the scar tissue that developed has overlapped the borders of the original patch and the stitches, so no correct measurement could be done, but the final scar tissue was not smaller than the original size of the patches.

Histological analysis was carried out after hematoxylin-eosin and picrosirius staining by an experienced pathologist (Fig. 3). Initially, a fibrin layer appeared on the peritoneal surface of the patches, and then with moderate inflammatory reaction, granulation tissue developed on the same place. From this, an organization process started, with an increased number of fibroblasts and fibrocytes, and neovascularization. The new capillaries had invaded the internal part of the patches from the diaphragm, while on the other peritoneal surface, a thin cellular layer had developed, which is a sign of peritoneal integration. This altogether formed a thicker scar tissue incorporating the implanted patches. Signs of

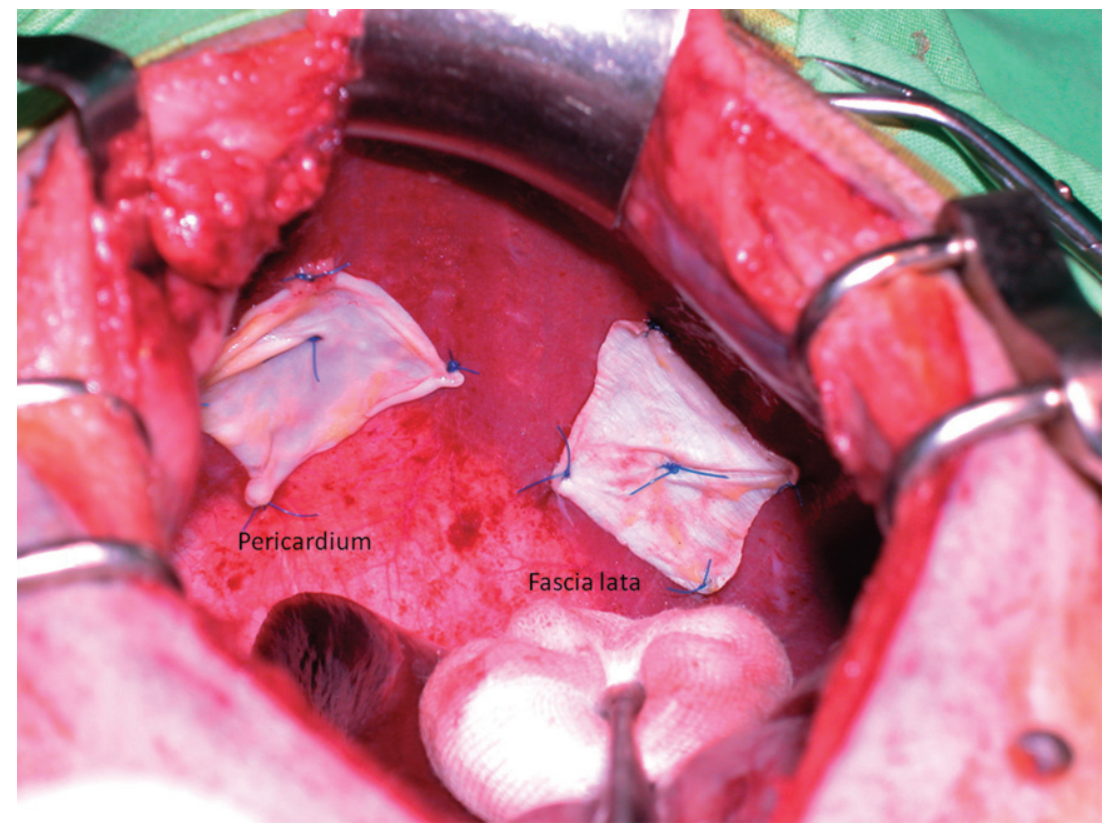

Fig. 1 Intraoperative view of the patches fixed to the diaphragm. 


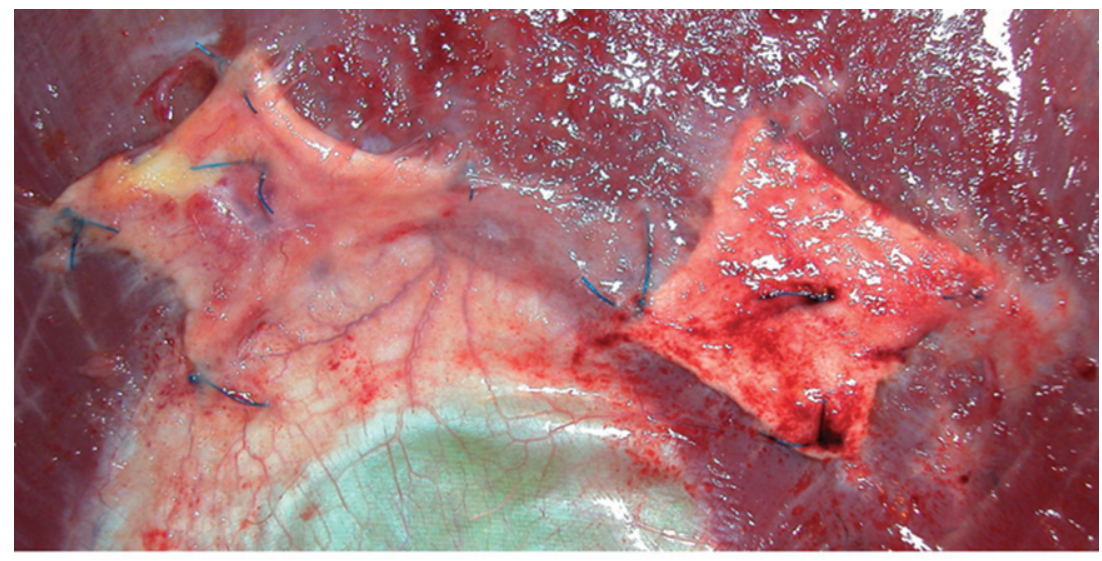

Patches after 1 month

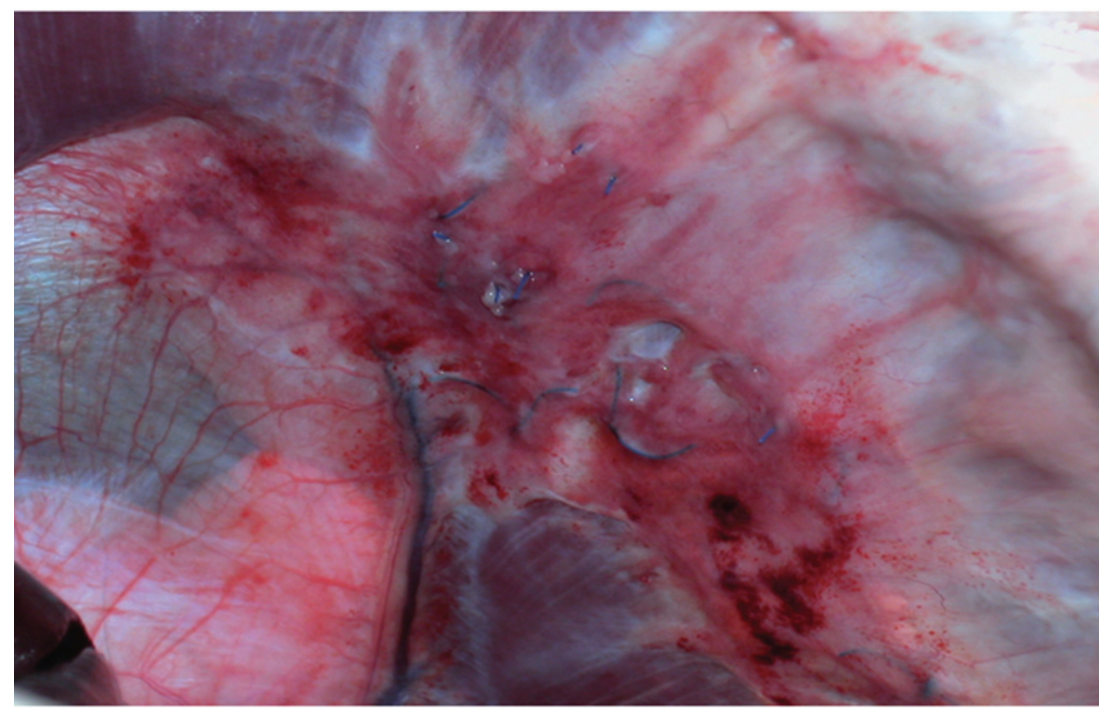

Patches after 6 months

Fig. 2 Macroscopic view of the patches after 1 and 6 months.

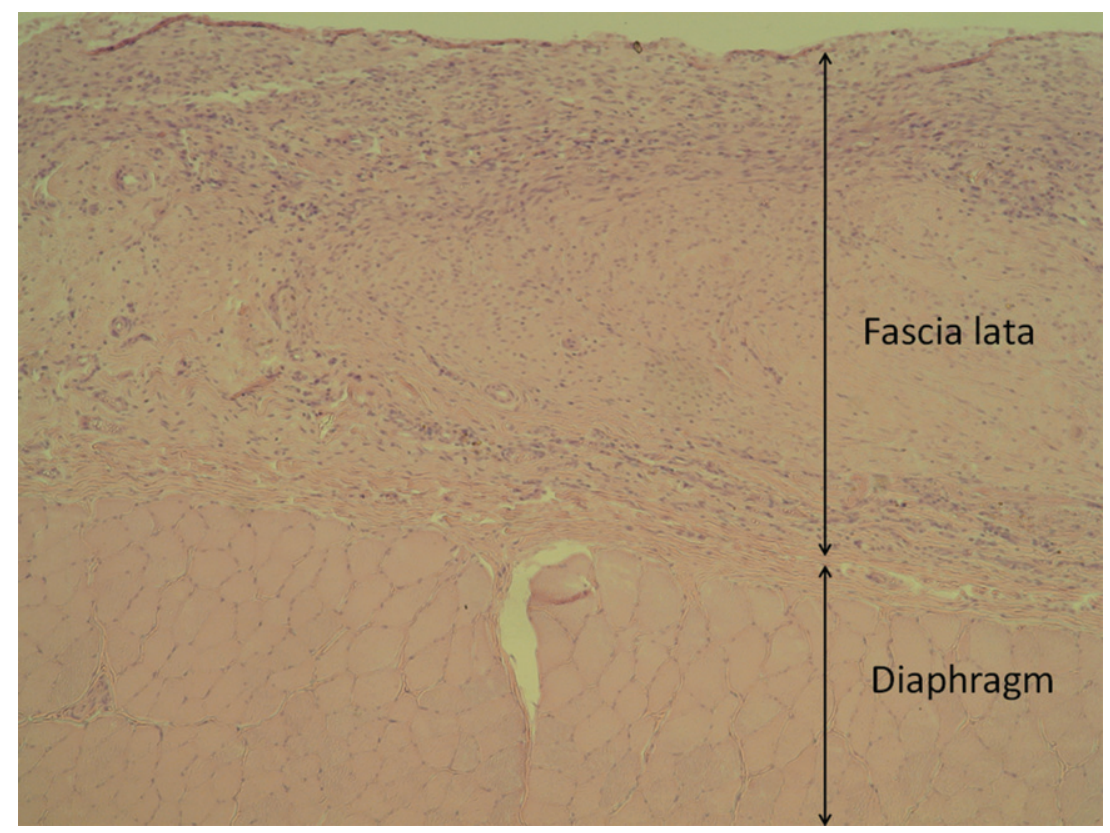

Fig. 3 Microscopic view of a fascia lata patch after 6 months with complete integration.

(C) 2011 Copyright the Authors

Journal compilation (C 2011, Wiley Periodicals, Inc. and the International Society for Diseases of the Esophagus 
abcedation or serious inflammation were also not detected upon microscopic examination.

\section{DISCUSSION}

The reliable reconstruction of large-sized hiatal hernias is not yet resolved. The literature suggests many different methods with equal advantages and disadvantages. Some authors are satisfied with the direct closure of the gap and report on good longterm results. ${ }^{4}$ However, comparing different series, the rate of recurrence is generally higher in cases of not reinforced reconstructions. ${ }^{1}$ This is why most of the authors recommend some kind of reinforcement.

Regarding the method of closure, the crura can be sutured directly, which may create tension on the stitches. To reinforce this, an onlay mesh should be applied to cover the suture line with 1-2 cm overlap. Another option is the tension-free repair when no direct closure is carried out, but a mesh is fixed to the sides of the gap, again with proper overlap as by ventral hernia repair. ${ }^{5}$

There is also a choice considering the material of the patches which can be either biologic or artificial. These methods use differently shaped meshes of several components, such as polypropylene, polyester, polytetrafluoroethylene (PTFE), or their combinations. ${ }^{6}$ Although these artificial materials generate proper scar tissue, they may also densely adhere to the wall of the alimentary tract, causing dysphagia or even penetration, which is a serious complication. ${ }^{7}$ Patches made from PTFE adhere less, but with this dislocation, rates increase. Another disadvantage of these meshes is their cost, which can be pretty high regarding PTFE or composite types. There are also biologic implants prepared from animal tissue, which cause less complication, but are also very expensive. Due to these data, we tried to find a cheap biologic reinforcement method and introduced the teres ligament plasty into clinical practice. ${ }^{2}$ It has many advantages, but, unfortunately, it is not applicable in every patient because of anatomical variations. Thanks to the different reinforcement strategies, the rate of hiatal hernia recurrence dropped to $0-14 \%{ }^{8}$

It is accepted for a long time that transferred connective tissue islets can cause permanent scar tissue formation at their new location. Such methods are used in neuro-, heart, orthopedic, thorax, urologic, or gynecologic surgery. ${ }^{3,9,10}$ There are approved protocols for tissue preservation and conservation. Based on these data, our experiment aimed a new utilization of cryopreserved connective tissue transplants.

The procedure was quite simple; there were no adverse reactions and the transplants did not resolve either. They organized and were incorporated in a new scar tissue, increasing tissue thickness, thus enhancing strength. Because of the shrinkage, which is common with all meshes, the size of the patch has to be larger than the area covered. This is also the accepted rule at ventral hernia repair. If the fixation is good, there is no danger of later dislocation due to the integration into the host tissue. There is also no danger of penetration after the organization is complete.

In those departments where transplantation surgery is performed, cryopreservation of the pericardium could be a good option for later connective tissue reinforcement. We do not think there is a significant difference between preserved and autologous patches regarding their scar generating properties. Where both possibilities are available, patients should be offered to choose based on the mentioned advantages and disadvantages. We found no data in the literature regarding disease transfer with preserved tissue grafts, and considering the agents used at the preservation, this danger is negligible; however, $100 \%$ safety cannot be guaranteed. With the active tissue bank at our institute, the only extra costs for the cryopreservation were the agents that we used. Compared with the costs of the purchased biologic meshes, these were significantly less. Where this possibility is not given, or there is a fear of transplantation of foreign tissue, a fascia lata patch from the same patient could be the preferred choice. Possible disadvantages in that case could be the scar on the thigh and the slight protrusion of the muscle where the fascia was resected. Fascia lata transfer has already been used at our department of thoracic surgery for the reinforcement of stapled lung resections, with good results. ${ }^{3}$ There were no complications at the fascia lata harvest site in those cases. At the cost of a 5- to 7-cm long incision on the lower thigh, a strong biologic patch from the own tissue can be harvested and transferred also to reinforce the hiatoplasty. We think that for human application, the autologous fascia lata reinforcement should be used as an onlay procedure at first, as the tension on the patch is weaker than that in a tension-free reconstruction. Ethics approval process for this has been started. Based on the experience of our thoracic department, a $7 \times 6 \mathrm{~cm}$ large patch can be harvested, which should be enough for an onlay hernia repair. Before it could be applied as a tension-free method to bridge a larger gap without primary closure, we think a further experimental study would be advisable to test the method under different circumstances.

\section{Acknowledgments}

Dr A. Vereczkei, Dr G. Varga, Dr T. Tornoczky, Dr A. Papp, and Dr Ö. P. Horvath have no conflicts of interest or financial ties to disclose.

\section{References}

1 Hashemi M, Peters J H, DeMeester T R et al. Laparoscopic repair of large type III hiatal hernia: objective followup 
reveals high recurrence rate. J Am Coll Surg 2000; 190: 53360.

2 Varga G, Cseke L, Kalmar K, Horvath O P. Laparoscopic repair of large hiatal hernia with teres ligament: midterm follow-up: a new surgical procedure. Surg Endosc 2008; 22: $881-4$.

3 Molnar T F, Rendeki S, Lukacs L, Horvath O P. Improvement of air tightness of stapled lung parenchyma using fascia lata. Interact Cardiovasc Thorac Surg 2003; 2: 5035.

4 Gouvas N, Tsiaoussis J, Athanasakis E, Zervakis N, Pechlivanides G, Xynos E. Simple suture or prosthesis hiatal closure in laparoscopic repair of paraesophageal hernia: a retrospective cohort study. Dis Esophagus 2011; 24: 69-78.

5 Basso N, Rosato P, De Leo A, Genco A, Rea S, Neri T. 'Tension-free' hiatoplasty, gastrophrenic anchorage, and 360 degree funoplication in the laparoscopic treatment of paraesophageal hernia. Surg Laparosc Endosc Percutan Tech 1999; 9: $257-62$.
6 Frantzides C T, Madan A K, Carlson M A, Stavropoulos G P. A prospective randomized trial of laparoscopic polytetrafluoroethylene (PTFE) patch repair vs simple cruroplasty for large hiatal hernia. Arch Surg 2002; 137: 649-52.

7 Stadlhuber R J, Sherif A E, Mittal S K et al. Mesh complications after prosthetic reinforcement of hiatal closure: a 28-case series. Surg Endosc 2009; 23: 1219-26.

8 Soricelli E, Basso N, Genco A, Cipriano M. Long-term results of hiatal hernia mesh repair and antireflux laparoscopic surgery. Surg Endosc 2009; 23: 2499-504.

9 Parízek J, Mĕricka P, Husek Z et al. Detailed evaluation of 2959 allogeneic and xenogeneic dense connective tissue grafts (fascia lata, pericardium, and dura mater) used in the course of 20 years for duraplasty in neurosurgery. Acta Neurochir (Wien) 1997; 139: 827-38.

10 Us M H, Sungun M, Sanioglu S et al. A retrospective comparison of bovine pericardium and polytetrafluoroethylene patch for closure of ventricular septal defects. J Int Med Res 2004; 32: 218-21. 